\title{
Article \\ Efficacy of Catheter Ablation for Atrial Arrhythmias in Patients with Arrhythmogenic Right Ventricular Cardiomyopathy-A Multicenter Study
}

\author{
Alessio Gasperetti ${ }^{1,2,3,4, *} \mathbb{0}$, Cynthia A. James ${ }^{4}$, Liang Chen ${ }^{5}$, Niklas Schenker ${ }^{6}$, Michela Casella ${ }^{2,7}$, \\ Shinwan Kany ${ }^{8}{ }^{-}$, Shibu Mathew ${ }^{6}$, Paolo Compagnucci ${ }^{2,3}{ }^{\circledR}$, Andreas Müssigbrodt ${ }^{9,10}$, Henrik K. Jensen ${ }^{11,12}$,

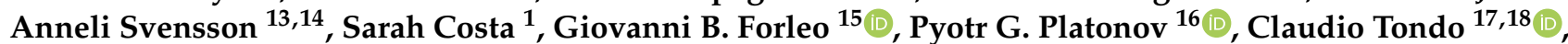 \\ Jiang-Ping Song ${ }^{5}$, Antonio Dello Russo ${ }^{2,3}$, Frank Ruschitzka ${ }^{1}{ }^{10}$, Corinna Brunckhorst ${ }^{1}$, Hugh Calkins ${ }^{4}$, \\ Firat Duru ${ }^{1,+}+\mathbb{C}^{5}$ and Ardan M. Saguner ${ }^{1,+}$
}

check for

updates

Citation: Gasperetti, A.; James, C.A.; Chen, L.; Schenker, N.; Casella, M.; Kany, S.; Mathew, S.; Compagnucci, P.; Müssigbrodt, A.; Jensen, H.K.; et al. Efficacy of Catheter Ablation for Atrial Arrhythmias in Patients with Arrhythmogenic Right Ventricular Cardiomyopathy-A Multicenter Study. J. Clin. Med. 2021, 10, 4962. https://doi.org/10.3390/jcm10214962

Academic Editors: Emmanuel Andrès and Luisa Mestroni

Received: 8 September 2021

Accepted: 17 October 2021

Published: 26 October 2021

Publisher's Note: MDPI stays neutral with regard to jurisdictional claims in published maps and institutional affiliations.

Copyright: (c) 2021 by the authors. Licensee MDPI, Basel, Switzerland. This article is an open access article distributed under the terms and conditions of the Creative Commons Attribution (CC BY) license (https:/ / creativecommons.org/licenses/by/ $4.0 /)$
1 Department of Cardiology, University Heart Center, University Hospital Zurich, Rämistrasse 100, 8091 Zurich, Switzerland; sarah.costa@usz.ch (S.C.); frank.ruschitzka@usz.ch (F.R.); Corinna.Brunckhorst@usz.ch (C.B.); firat.duru@usz.ch (F.D.); ardan.saguner@usz.ch (A.M.S.)

2 Cardiology and Arrhythmology Clinic, University Hospital "Ospedali Riuniti Umberto I-Lancisi-Salesi”, 60126 Ancona, Italy; michelacasella@hotmail.com (M.C.); paolocompagnucci1@gmail.com (P.C.); antonio.dellorusso@gmail.com (A.D.R.)

3 Department of Biomedical Science and Public Health, Marche Polytechnic University, 60126 Ancona, Italy

4 Division of Cardiology, Department of Medicine, Johns Hopkins Hospital, Baltimore, MD 21205, USA; cjames7@jhmi.edu (C.A.J.); hcalkins@jhmi.edu (H.C.)

5 State Key Laboratory of Cardiovascular Disease, Fuwai Hospital, Peking Union Medical College, Beijing 100000, China; chenliang@fuwaihospital.org (L.C.); fwsongjiangping@126.com (J.-P.S.)

6 Department of Cardiology, Asklepios Klinik St. Georg Hamburg, 20099 Hamburg, Germany; nik.schenker@asklepios.com (N.S.); dr.mathew.shibu@googlemail.com (S.M.)

7 Department of Clinical, Special and Dental Sciences, Marche Polytechnic University, 60126 Ancona, Italy

8 Department of Cardiology, University Heart and Vascular Centre Hamburg, University Medical Centre Hamburg-Eppendorf, 20251 Hamburg, Germany; s.kany@uke.de

9 Department of Electrophysiology, Heart Center University of Leipzig, 04289 Leipzig, Germany; andreas.muessigbrodt@gmail.com

10 Department of Cardiology, University Hospital of Martinique, 97200 Fort de France, Martinique, France

11 Department of Cardiology, Aarhus University Hospital, 8200 Aarhus, Denmark; hkjensen@clin.au.dk

12 Department of Clinical Medicine, Health, Aarhus University, 8200 Aarhus, Denmark

13 Department of Cardiology, Linköping University, 58183 Linköping, Sweden; anneli.svensson@gmail.com

14 Department of Medical and Health Sciences, Linköping University, 58183 Linköping, Sweden

15 Dipartimento di Cardiologia, ASST FBF-Sacco, 20149 Milano, Italy; forleo@me.com

16 Lund University Arrhythmia Clinic, Department of Cardiology, Skåne University Hospital, 22185 Lund, Sweden; pyotr.platonov@med.lu.se

17 Heart Rhythm Center, Centro Cardiologico Monzino, IRCCS, 20138 Milan, Italy; claudio.tondo@ccfm.it

18 Department of Clinical Sciences and Community Health, University of Milan, 20122 Milan, Italy

* Correspondence: alessio.gasperetti93@gmail.com

+ Saguner and Duru share last co-authorship.

Abstract: Background: Atrial arrhythmias are present in up to $20 \%$ of patients with arrhythmogenic right ventricular cardiomyopathy (ARVC). Catheter ablation (CA) is an effective treatment for atrial arrhythmias in the general population. Data regarding CA for atrial arrhythmias in ARVC are scarce. Objective: To assess the safety and efficacy of CA for atrial arrhythmias in patients with ARVC. Methods: In this international collaborative effort, all patients with a definite diagnosis of ARVC undergoing CA for atrial fibrillation (AF), focal atrial tachycardia (AT), or cavotricuspid isthmus (CTI)-dependent atrial flutter (AFl) were extracted from twelve ARVC registries. Demographic, periprocedural, and long-term arrhythmic outcome data were collected. Results: Thirty-seven patients were enrolled in the study (age $50.2 \pm 16.6$ years, male $84 \%$, $\mathrm{CHA}_{2} \mathrm{DS}_{2}$ VASc $1(1,2)$, HASBLED 0 (0-2)). The arrhythmia leading to CA was AF in $23(62 \%)$, focal left AT in 5 (14\%), and CTI-dependent AFl in 9 (24\%). Acute procedural success was achieved in all procedures but one ( $n=1$ focal left AT; 97\% acute success). The median follow-up period was 27 (13-67) months, and 96\%, $74 \%$, and $61 \%$ of patients undergoing AF ablation were free from any atrial arrhythmia recurrence 
after a single procedure at 6 months, 12 months, and last follow-up, respectively. After focal AT ablation, freedom from atrial arrhythmia recurrence was $80 \%, 80 \%$, and $60 \%$ at 6 months, 12 months, and last follow-up, respectively. All patients undergoing CTI ablation were free from atrial arrhythmia recurrences at 6 months, with $89 \%$ single-procedural arrhythmic freedom at last follow-up. One major complication (2.7\%; PV stenosis requiring PV stenting) occurred. Conclusions: CA is safe and effective in managing atrial arrhythmias in patients with ARVC, with success rates comparable to the general population.

Keywords: arrhythmogenic right ventricular cardiomyopathy; atrial fibrillation; atrial flutter; pulmonary vein isolation; ablation in special populations

\section{Introduction}

Arrhythmogenic right ventricular cardiomyopathy (ARVC) is an inherited cardiomyopathy characterized by a progressive myocardial fibro-fatty infiltration (FFI), predominantly originating in the right ventricle (RV) [1]. Although ventricular dysfunction and ventricular arrhythmias represent the hallmarks of ARVC, a growing body of evidence from both ARVC animal models and patient cohorts has indicated atrial involvement [2-4]. The atria of patients with ARVC have been shown to be dilated and their function impaired, with the right atrium (RA) being predominantly affected by this process [3-5]. Considering electrocardiographic features, Platonov PG et al. first described an altered electrical conduction within the atria of patients with ARVC [6], and subsequent cohort studies reported a high rate of atrial arrhythmias in ARVC [3,7,8]. Particularly, atrial fibrillation (AF) and atrial flutter $(\mathrm{AFl})$ were shown to be more frequent and presented at a younger age in patients with ARVC as compared to the general population [7,9].

Catheter ablation (CA) is an effective therapy for the treatment of atrial arrhythmias in the general population [10,11]. Data regarding the use, safety, and efficacy of CA in the management of atrial arrhythmias in ARVC are scarce, and previous work is limited to a few small sample-sized reports [12,13]. Therefore, the aim of this international multicenter study was to analyze the efficacy and safety of CA for the treatment of atrial arrhythmias in patients with ARVC.

\section{Methods}

The ARVC registries at 12 tertiary care institutions (University Hospital Zurich, Switzerland; Johns Hopkins Medical University, USA; IRCCS Centro Cardiologico Monzino, Italy; Asklepios Clinic St. George, Hamburg, Germany; Fuwai University Hospital, Beijing, China; Aarhus Hospital, Denmark; Skane University Hospital, Sweden; Linkoeping University Hospital, Sweden; Azienda Ospedaliera Luigi Sacco, Italy; University Clinic Hamburg-Eppendorf, Germany; University Hospital of Ancona, Italy; Heart Center University of Leipzig, Germany) were retrospectively searched for all consecutive ARVC patients fulfilling the inclusion criteria of the study. The earliest patient enrolled had his atrial procedure performed in 2014, while the last patient enrolled had her procedure performed in 2020.

The study was approved by the ethical boards of each contributing center, and informed consent was retrieved at each center in accordance with local and national regulations. This study was performed in accordance with the Declaration of Helsinki. The data that support the findings of this study are available from the corresponding author upon reasonable request.

\subsection{Study Inclusion Criteria}

Patients were enrolled in the present study upon the following inclusion criteria: 
- An ARVC diagnosis in accordance with the 2010 International Task Force Criteria (ITFC) [14]. Only patients reaching a "definite" diagnosis were included in the final cohort.

- Performance of CA for an atrial arrhythmia of interest at any point during the clinical course; atrial arrhythmias of interest were AF, left-sided atrial tachycardia (AT), and CTI-dependent AFl. No ablation strategy or ablation energy source was pre-specified for study enrollment.

- A minimum follow-up time of 6 months, with at least one follow-up visit with an arrhythmic assessment after CA.

No additional exclusion criteria were used.

Of note, the present study was aimed to assess the effectiveness of catheter ablation for left-sided atrial arrhythmias including AF in patients with ARVC. CTI-dependent AFI patients were also included due to it being the most common atrial arrhythmia in patients with ARVC and its common association with AF. Data of patients with right-sided atrial tachycardia other than CTI-dependent AFl, due to its rarity and relatively low clinical yield in this patient population, were not collected.

\subsection{Data Collection}

All available demographics, baseline, and pre-, peri- and post-procedural data ( $<7$ days from the time of the procedure) were collected from patient medical records. Cardiac dimensions and function were retrieved, including data from echocardiography and cardiac magnetic resonance imaging, if available. If an adequate comparability in the obtained data was available, numerical values were reported. In the case of noncomparability, data were presented using a semiquantitative description (e.g., right/left atrium dimension: "normal", "moderate dilation", "severe dilation"). Imaging data were acquired according to current guidelines and/or local practice. Only membrane-active anti-arrhythmic drugs (Class I and Class III of the Vaughan Williams classification) were considered anti-arrhythmic drugs for the purpose of this study. Arrhythmic follow-up was assessed using 24 h, 48 h, and 7-day Holter ECGs; implantable loop recorders; or remote/in-person implantable cardioverter defibrillator (ICD) interrogation based on the clinical practice and expertise of each center. An ICD was available in $76 \%$ of the population for arrhythmic follow-up assessment and represented the most employed methodology. All data were pseudonymized and collected in a centralized database.

\subsection{Study Definitions, Aims, and Outcomes}

Acute success was defined as follows: (a) achievement of pulmonary vein isolation (PVI) in PVI-based procedures; (b) arrhythmia termination, if present at the time of CA, and / or non-inducibility of atrial arrhythmias in left-sided non-PVI-based procedures at the end of the procedure; (c) achievement of bidirectional block in CTI ablation procedures. Long-term success was defined as the absence of atrial arrhythmia recurrence during followup. An atrial arrhythmia recurrence at follow-up was defined as any atrial arrhythmia episode lasting $>30 \mathrm{~s}$ after a 3-month post-procedural blanking period $+/-$ antiarrhythmic medication.

The aim of this study was to assess the efficacy and safety of CA for the treatment of atrial arrhythmias in patients with ARVC. The primary outcome of the study was freedom from atrial arrhythmia recurrence at 6 months. Secondary outcomes included mid-term (12-month follow-up) and long-term (last available follow-up) outcome data as well as periprocedural complication rate and changes in pharmacological therapy in the long term.

\subsection{Statistical Analysis}

All statistical analyses were performed using STATA v. 14.0 (STATACorp, College Station, TX, USA).

Distribution of all continuous variables was tested for normality using the ShapiroWilk test. Normally distributed variables are reported as mean \pm standard deviation, while 
non-normally distributed data are reported as median (with interquartile ranges (IQR)). Categorical variables are expressed as counts (percentage). Kaplan-Meier estimates were used to report arrhythmic outcome data.

\section{Results}

\subsection{Patient Population}

A total of 37 patients with definite ARVC ( 32 probands and 5 family members) fulfilling the inclusion criteria were enrolled in this study. ARVC diagnosis was reached at a mean age of $50.2 \pm 16.6$ years. ARVC diagnostic characteristics are shown in Table 1.

Table 1. ARVC diagnostic characteristics of the study cohort.

\begin{tabular}{|c|c|}
\hline \multicolumn{2}{|c|}{ ARVC Diagnostic Characteristics } \\
\hline Age at ARVC diagnosis (years), mean \pm s.d. & $50.2 \pm 16.6$ \\
\hline Sustained VAs before/at ARVC diagnosis, $n(\%)$ & $24(65)$ \\
\hline ARVC score, median [IQR] & $5(4-6)$ \\
\hline \multicolumn{2}{|l|}{ Category I-Global or regional dysfunction } \\
\hline Major, $n(\%)$ & $20(54)$ \\
\hline Minor, $n(\%)$ & $11(30)$ \\
\hline \multicolumn{2}{|l|}{ Category II-Tissue characterization of the wall } \\
\hline Major, $n(\%)$ & $4(11)$ \\
\hline Minor, $n(\%)$ & $3(8)$ \\
\hline \multicolumn{2}{|l|}{ Category III-Repolarization abnormalities } \\
\hline Major, $n(\%)$ & $13(35)$ \\
\hline Minor, $n(\%)$ & $9(26)$ \\
\hline \multicolumn{2}{|l|}{ Category IV-Depolarization abnormalities } \\
\hline Major, $n(\%)$ & $10(27)$ \\
\hline Minor, $n(\%)$ & $12(32)$ \\
\hline \multicolumn{2}{|l|}{ Category V-Ventricular arrhythmias } \\
\hline Major, $n(\%)$ & $19(51)$ \\
\hline Minor, $n(\%)$ & $16(43)$ \\
\hline \multicolumn{2}{|l|}{ Category VI-Family history } \\
\hline Major, $n(\%)$ & $3(8)$ \\
\hline Minor, $n(\%)$ & $4(11)$ \\
\hline Genetic testing performed, $n(\%)$ & $13(35)$ \\
\hline Negative, $n(\%)$ & $6(46)^{*}$ \\
\hline Positive, $n(\%)$ & $7(54) *$ \\
\hline PKP-2, $n(\%)$ & $5(71) *$ \\
\hline DSG-2, $n(\%)$ & $2(29) *$ \\
\hline
\end{tabular}

DSG-2: desmoglein-2; IQR: interquartile range; PKP-2: plakophilin-2; VA: ventricular arrhythmias. * Relative percentage calculated using as denominator the number of available data.

The atrial arrhythmia treated by CA was AF in $23(62 \%)$ patients, left-sided focal AT in $5(14 \%)$ patients, and CTI-dependent AFl in $9(24 \%)$ patients, respectively. The first atrial arrhythmia episode was observed at a mean age of $48.7 \pm 15.3$ years, and in most cases, its presentation preceded the diagnosis of ARVC (median difference between atrial arrhythmia presentation and ARVC diagnosis: $-2(-4 ; 0)$ years). Baseline characteristics are summarized in Table 2.

\subsection{Periprocedural Data}

Patients underwent CA less than 1 year (0-2) after their first atrial arrhythmia episode (mean age at CA: $49.0 \pm 15.4$ years). Three patients $(8 \%)$ were referred for CA after an inappropriate ICD discharge that was triggered by an atrial arrhythmia. Data for RA and left atrial (LA) dimensions were available for $30(81 \%)$ and $32(86 \%)$ patients, respectively. The majority of patients $(29 / 32)$ had a normal LA size, while moderate or severe RA dilation was observed in $43 \%$ of cases. 
Table 2. Baseline clinical characteristics for the study cohort.

\begin{tabular}{|c|c|}
\hline \multicolumn{2}{|c|}{ Baseline Characteristics (Overall Cohort, $n=37$ ) } \\
\hline Male, $n(\%)$ & $31(84)$ \\
\hline Age at first atrial arrhythmia presentation (years), mean \pm s.d. & $48.7 \pm 15.3$ \\
\hline \multicolumn{2}{|l|}{ First atrial arrhythmia type } \\
\hline Atrial fibrillation, $n(\%)$ & $23(62)$ \\
\hline Paroxysmal, $n(\%)$ & $16(43)$ \\
\hline Persistent, $n(\%)$ & $5(14)$ \\
\hline Long-standing persistent, $n(\%)$ & $2(5)$ \\
\hline Focal left-sided atrial tachycardia, $n(\%)$ & $5(14)$ \\
\hline Cavotricuspid isthmus-dependent atrial flutter, $n(\%)$ & $9(24)$ \\
\hline Probands, $n(\%)$ & $32(87)$ \\
\hline Athletes, $n(\%)$ & $7(19)$ \\
\hline $\operatorname{BSA}\left(\mathrm{m}^{2}\right)$, median $[\mathrm{IQR}]$ & $1.9(1.8-2.1)$ \\
\hline CHA2DS2VASc, median [IQR] & $1(1,2)$ \\
\hline Age $>64$ y.o., $n(\%)$ & $7(19)$ \\
\hline Age $>74$ y.o., $n(\%)$ & $2(5)$ \\
\hline History of CHF, $n(\%)$ & $15(41)$ \\
\hline Female gender, $n(\%)$ & $6(16)$ \\
\hline Hypertension, $n(\%)$ & $12(32)$ \\
\hline Previous stroke/TIA, $n(\%)$ & $3(8)$ \\
\hline Vascular disease history, $n(\%)$ & $4(11)$ \\
\hline Diabetes, $n(\%)$ & $3(8)$ \\
\hline HAS-BLED, median [IQR] & $1(0-2)$ \\
\hline Age $>65$ y.o, $n(\%)$ & $9(24)$ \\
\hline Hypertension, $n(\%)$ & $12(32)$ \\
\hline Renal disease, $n(\%)$ & $4(11)$ \\
\hline Liver disease, $n(\%)$ & 0 \\
\hline Stroke history, $n(\%)$ & $3(8)$ \\
\hline Prior major bleeding, $n(\%)$ & $1(3)$ \\
\hline Labile INR, $n(\%)$ & $1(3)$ \\
\hline Medication predisposing bleeding, $n(\%)$ & $14(38)$ \\
\hline Alcohol abuse, $n(\%)$ & $3(8)$ \\
\hline EHRA score, median [IQR] & $1(0-2)$ \\
\hline $\mathrm{ICD}, n(\%)$ & $28(76)$ \\
\hline Inappropriate discharge due to atrial arrhythmia, $n(\%)$ & $3(8)$ \\
\hline
\end{tabular}

BSA: body surface area; CHF: congestive heart failure; ICD: implantable cardioverter-defibrillator; INR: international normalized ratio.

\subsection{Procedural Data}

A total of 23 (62\%) ARVC patients underwent PVI. In 16 (43\%) of them, only circumferential PVI was performed. The other seven patients had PVI plus additional ablation targets: in four cases (11\%), an anterior mitral isthmus line was performed; in two cases $(5 \%)$, posterior wall isolation was performed; and in one case $(3 \%)$, a roof line was additionally performed. In six patients undergoing PVI, CTI ablation was additionally performed. In $22(96 \%)$ patients with AF, radiofrequency energy was used for PVI, while cryoballoon was used in 1 patient (4\%). Five patients (14\%) underwent left-sided atrial ablation (focal ablation in four and anterior mitral isthmus line in one) without PVI, while in the remaining nine patients $(24 \%)$, CTI ablation was performed.

\subsection{Acute Procedural Data}

Acute procedural success was achieved in 36 of 37 patients (97\%). Catheter ablation failed in one patient with a focal left-sided AT. Six procedures required an intra-operative electrical cardioversion. Two periprocedural complications (5.4\%) were encountered: a left inferior PV stenosis (after PVI) requiring subsequent stenting in one patient was considered a major complication, whereas a groin hematoma after AFl ablation in another patient, which was managed conservatively without further sequelae, was considered a minor complication. Periprocedural and procedural data are reported in Table 3. 
Table 3. Periprocedural and procedural characteristics of the study cohort.

\begin{tabular}{|c|c|}
\hline \multicolumn{2}{|c|}{ Periprocedural and Procedural Characteristics } \\
\hline Age at CA (years), mean \pm s.d. & $49.0 \pm 15.4$ \\
\hline \multicolumn{2}{|l|}{ Pharmacological therapy at the time of CA } \\
\hline Oral anticoagulation, $n(\%)$ & $23(62)$ \\
\hline VKA, $n(\%)$ & $10(27)$ \\
\hline DOAC, $n(\%)$ & $13(35)$ \\
\hline Anti-arrhythmic therapy, $n(\%)$ & $11(30)$ \\
\hline Class Ic, $n(\%)$ & $3(8)$ \\
\hline Class III, $n(\%)$ & $8(22)$ \\
\hline \multicolumn{2}{|l|}{ Morphology and functional data at the time of CA } \\
\hline Right atrial dimension available, $n(\%)$ & $30(81)$ \\
\hline Normal dimension, $n(\%)$ & $17(57) *$ \\
\hline Moderate dilation, $n(\%)$ & $9(30) *$ \\
\hline Severe dilation, $n(\%)$ & $4(13) *$ \\
\hline Left atrial dimension available, $n(\%)$ & $32(86)$ \\
\hline Normal dimension, $n(\%)$ & $29(91) *$ \\
\hline Moderate dilation, $n(\%)$ & $1(3) *$ \\
\hline Severe dilation, $n(\%)$ & $2(6) *$ \\
\hline RVEF available, $n(\%)$ & $21(57)$ \\
\hline Normal (>49\%), $n(\%)$ & $4(19) *$ \\
\hline Reduced $(26-49 \%), n(\%)$ & $15(71) *$ \\
\hline Severely reduced $(<26 \%), n(\%)$ & $2(10) *$ \\
\hline $\operatorname{LVEF}(\%)$, mean \pm s.d. & $52.4 \pm 6.8$ \\
\hline \multicolumn{2}{|l|}{ Area targeted during CA } \\
\hline Pulmonary vein isolation, $n(\%)$ & $23(62)$ \\
\hline PVI only, $n(\%)$ & $16(43)$ \\
\hline PVI + anterior mitral line, $n(\%)$ & $4(11)$ \\
\hline PVI + posterior wall isolation, $n(\%)$ & $2(5)$ \\
\hline PVI + roof line, $n(\%)$ & $1(3)$ \\
\hline Non-PVI-based left-sided procedures, $n(\%)$ & $5(14)$ \\
\hline Focal ablation, $n(\%)$ & $4(11)$ \\
\hline Anterior mitral line, $n(\%)$ & $1(3)$ \\
\hline Stand-alone CTI ablation, $n(\%)$ & $9(24)$ \\
\hline Power settings for ablation, Watts [IQR] & $40(35-40)$ \\
\hline \multicolumn{2}{|l|}{ Procedural time } \\
\hline Left-sided procedure (min), median [IQR] & $140(105-143)$ \\
\hline Right-sided procedure (min), median [IQR] & $70(60-110)$ \\
\hline \multicolumn{2}{|l|}{ Fluoroscopy time } \\
\hline Left-sided procedure (min), median [IQR] & $18.5(11.7-19.5)$ \\
\hline Right-sided procedure (min), median [IQR] & $13.5(12.0-14.0)$ \\
\hline Acute Success, $n(\%)$ & $36(97)$ \\
\hline Pulmonary vein isolation, $n(\%)$ & $23(100)$ \\
\hline Non PVI-based left-sided procedure, $n(\%)$ & $4(80)$ \\
\hline Stand-alone CTI, $n(\%)$ & $9(100)$ \\
\hline Need for ECV during procedure, $n(\%)$ & $6(16)$ \\
\hline Complications, $n(\%)$ & $2(5.4)$ \\
\hline Vascular access hematoma, $n(\%)$ & $1(2.7)$ \\
\hline PV stenosis, $n(\%)$ & $1(2.7)$ \\
\hline
\end{tabular}

* Relative percentage calculated using the number of available data as denominator. CA: catheter ablation, CTI: cavotricuspid isthmus; DOAC: direct oral anticoagulants; ECV: electrical cardioversion; LA: left atrium; LVEF: left ventricular ejection fraction; PV: pulmonary vein; RA: right atrium; RVEF: right ventricular ejection fraction; VKA: vitamin K antagonists.

\subsection{Long-Term Outcome Data}

All patients were followed up for a minimum of 6 months. The median follow-up period of the cohort was 27 (13-67) months.

Figure 1 displays single-procedural freedom from atrial arrhythmia recurrence rates +/- AADs, stratified by procedure type at 12 months. At 6 -months follow-up, $96 \%$ of patients with AF undergoing PVI-based procedures were free from arrhythmic recurrences, while at 12 months, their freedom from atrial arrhythmias was $74 \%$. At last available follow- 
up, 25/37 patients (68\%) were free from arrhythmic recurrences, with 2 patients being on a previously failed AAD ( $n=2$ Class III). Of patients undergoing left-sided non-PVI-based procedures, $80 \%$ (four out of five) were free from recurrences at both the 6- and 12-month follow-up after a single procedure. At last available follow-up, $60 \%$ (three out of five) of the patients were free from atrial arrhythmias ( $n=1$ patient on a class Ic AAD). Among the nine patients who underwent ablation of CTI-dependent AFl, $100 \%$ (nine out of nine) were free of recurrences at 6 months and $89 \%$ (eight out of nine) at 12 months and last follow-up after a single procedure ( $n=2$ patients on a class III AAD).

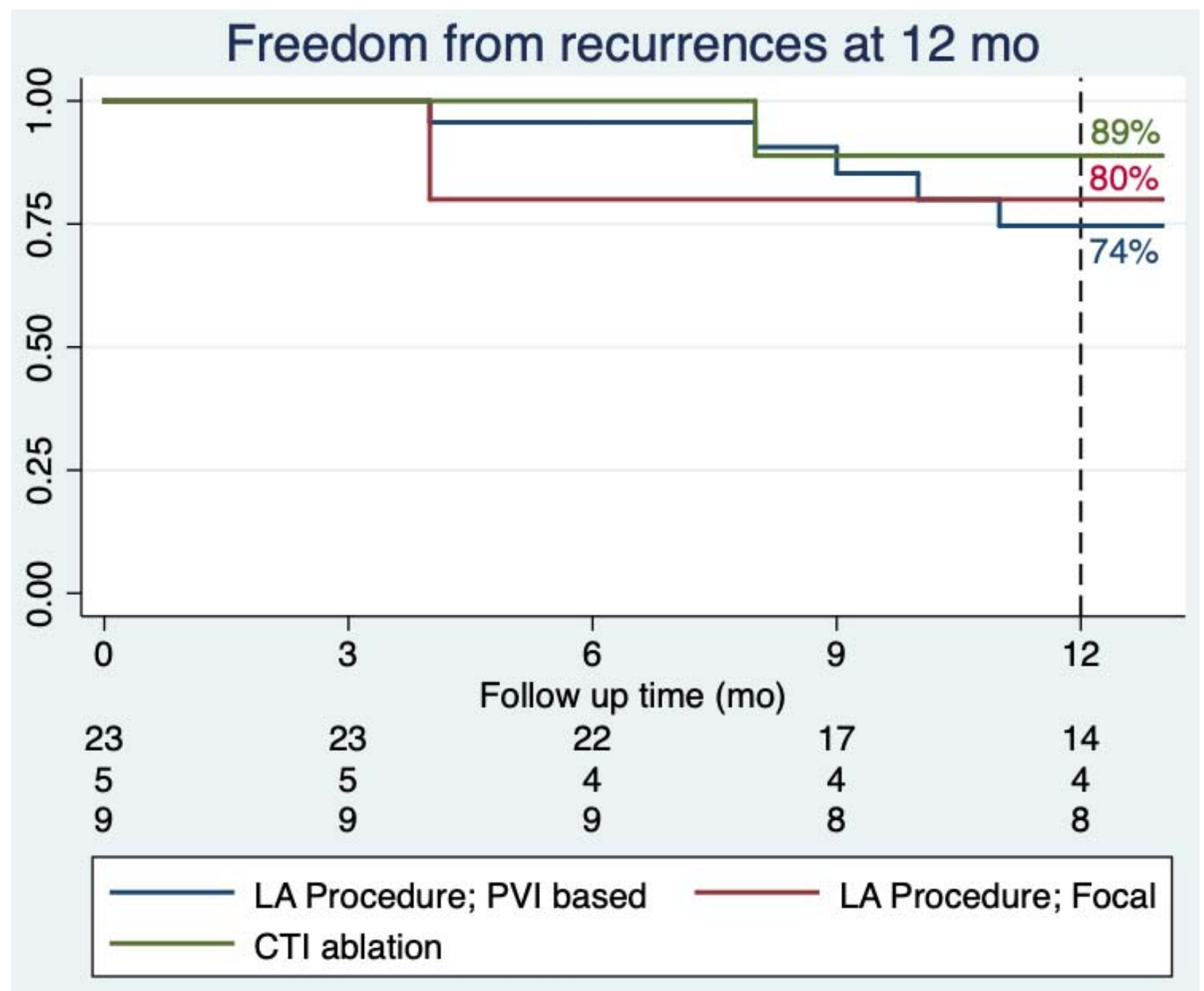

Figure 1. Single-procedural freedom from atrial arrhythmia recurrences stratified by ablation procedure performed at 12 months. CTI: cavotricuspid isthmus; LA: left atrial; PVI: pulmonary vein isolation.

During follow-up, a total of seven patients $(19 \%)$ underwent a repeat procedure ( $n=1$ for CTI-dependent AFl, $n=1$ for left-sided focal AT, and $n=5$ for AF). When including repeat procedures, all patients with CTI-dependent AFl were free from any atrial arrhythmia at last follow-up. At last follow-up, $24 \%$ and $46 \%$ of the population were on anti-arrhythmic drugs and on oral anticoagulation, respectively (no significant changes from baseline, $p=0.56$ and $p=0.12$, respectively). No severe bleeding events or cerebral vascular events were observed. The overall outcome data are summarized in Table 4 . 
Table 4. Cohort outcomes at follow up.

\begin{tabular}{|c|c|}
\hline \multicolumn{2}{|c|}{ Outcome Analysis } \\
\hline Follow-up time (months), median [IQR] & $27(13-67)$ \\
\hline \multicolumn{2}{|l|}{ Single-procedural freedom from any atrial arrhythmia } \\
\hline At 6 months, $n(\%)$ & $35 / 37(95)$ \\
\hline After PVI, $n(\%)$ & $22 / 23(96)$ \\
\hline After non-PVI-based left-sided procedure, $n(\%)$ & $4 / 5(80)$ \\
\hline After CTI ablation, $n(\%)$ & $9 / 9(100)$ \\
\hline At 12 months, $n(\%)$ & $26 / 33(79)$ \\
\hline After PVI, $n(\%)$ & $14 / 19(74)$ \\
\hline After non-PVI-based left-sided procedure, $n(\%)$ & $4 / 5(80)$ \\
\hline After CTI ablation, $n(\%)$ & $8 / 9(89)$ \\
\hline At last available follow-up, $n(\%)$ & $25 / 37(68)$ \\
\hline After PVI, $n(\%)$ & $14 / 23(61)$ \\
\hline After non-PVI-based left-sided procedure, $n(\%)$ & $3 / 5(60)$ \\
\hline After CTI ablation, $n(\%)$ & $8 / 9(89)$ \\
\hline \multicolumn{2}{|l|}{ Pharmacological therapy at last available follow-up } \\
\hline Oral anticoagulation, $n(\%)$ & $17(46)$ \\
\hline VKA, $n(\%)$ & $8(22)$ \\
\hline $\mathrm{DOAC}, n(\%)$ & $9(24)$ \\
\hline Anti-arrhythmic therapy, $n(\%)$ & $9(24)$ \\
\hline Class $\mathrm{Ib}, n(\%)$ & $1(3)$ \\
\hline Class Ic, $n(\%)$ & $5(14)$ \\
\hline Class III, $n(\%)$ & $3(8)$ \\
\hline LVEF at last available follow-up, mean \pm standard deviation & $49.4 \pm 12.4$ \\
\hline Major bleeding during follow-up, $n(\%)$ & 0 \\
\hline Stroke/TIA during follow-up, $n(\%)$ & 0 \\
\hline Inappropriate ICD shock due to atrial arrhythmias at follow-up, $n(\%)$ & 0 \\
\hline Patients undergoing a repeat procedure, $n(\%)$ & $7(19)$ \\
\hline PVI, $n(\%)$ & $3(8)$ \\
\hline Anterior mitral line, $n(\%)$ & $2(5)$ \\
\hline Focal ablation, $n(\%)$ & $1(3)$ \\
\hline CTI ablation, $n(\%)$ & $1(3)$ \\
\hline
\end{tabular}

\section{Discussion}

This study represents the largest systematic assessment of the efficacy and safety of CA for the treatment of atrial arrhythmias inpatients with ARVC to date.

The main findings of this international multicenter study are: (a) CA was an effective strategy for the management of atrial arrhythmias in patients with ARVC, with high acute procedural success and low complication rates; (b) CA achieved considerable longterm freedom from atrial arrhythmia recurrences, both for PVI and non-PVI-based ablative procedures; (c) at the time of CA, the majority of patients with ARVC and atrial arrhythmias presented with normal LA dimensions, whereas RA dimensions were increased in more than one-third.

\subsection{Atrial Arrhythmias and ARVC}

Up to $15-20 \%$ of ARVC patients enrolled in large and well-characterized cohorts were reported to suffer from episodes of atrial arrhythmias, with AF being the most common one $[3,7,8]$. Atrial arrhythmias in these patients may develop as part of a primary pathogenic process (atrial cardiomyopathy) due to desmosomal/connexome impairment in the atria or as a consequence of atrial dilation following depressed ventricular function and dilation.

The presence of atrial arrhythmias in patients with ARVC has important clinical consequences, such as an increased risk of inappropriate ICD discharges [3,15]. Of the 35 ARVC patients with atrial arrhythmias and an ICD reported by Chu et al. [12], 3 presented with inappropriate ICD therapies triggered by an atrial arrhythmia episode. A similar rate $(8 \%)$ of atrial arrhythmia-triggered inappropriate shocks was also found in our cohort, 
representing an important indication for the referral for CA in these patients. Additionally, the presence of atrial arrhythmias (AF in particular) has been strongly associated with an increase in life-threatening ventricular arrhythmic events in a study from Mazzanti et al. [16]. Although most of the attention in this specific subset of patients has been paid to ventricular arrhythmias, an adequate clinical management of patients with ARVC undoubtedly also requires an appropriate management of atrial arrhythmias.

\subsection{Outcomes of Catheter Ablation of Atrial Arrhythmias in ARVC}

$\mathrm{CA}$ is an effective and safe therapeutic option for the management of atrial arrhythmias in the general population [11]. To the best of our knowledge, prior evidence regarding the safety and efficacy of CA for atrial arrhythmias in patients with ARVC is limited to two small-scale studies. Chu et al. [12] reported feasibility data from 7 patients with ARVC suffering from CTI-dependent AFl undergoing CA for rhythm control, while CardonaGuarache et al. reported safety and feasibility of AA ablation in 11 patients $(n=3$ PVI; $n=2$ atypical flutter; $n=5$ typical flutter; $n=1 \mathrm{AT}$ ).

In our study, 23 ARVC patients underwent PVI and/or extended procedures for the management of AF/left-sided AT. Patients responded well to this intervention, achieving similar single-procedural rates of freedom from AF/left-sided AT as those reported in the general population. The rates of freedom from atrial arrhythmias recurrence at 6-month, 12-month, and at last available follow-up were $96 \%, 74 \%$, and $68 \%$, respectively. The predominance of paroxysmal AF, the normal LA size (in 91\%) and preserved LVEF, and the relatively young age at the time of CA probably represented factors positively influencing outcome. Five patients underwent CA for a focal, left-sided AT, showing $60 \%$ singleprocedural freedom from arrhythmias at last follow-up. Nine patients underwent CTI ablation for typical AFl. Although a dilated RA was present in $43 \%$ of the study population, CTI ablation was also very effective in this population, with eight out of nine patients being free from atrial arrhythmias after a single procedure at last follow-up. The ninth patient required repeat CTI ablation, after which no more atrial arrhythmias were observed. Complication rates except for PV stenosis (2.7\%) were low and similar to those reported in the general population referred for CA of atrial arrhythmias [17]. The rather high rate of PV stenosis was probably related to the small sample size of the overall population.

Our data suggest that patients with ARVC should not be precluded from CA for atrial arrhythmias in light of their underlying cardiac disease and that the management of AF/AFl should be similar to that offered to the general population. These findings could additionally result in particular interest for two reasons.

First, since atrial arrhythmias are associated with inappropriate ICD discharges and adverse outcomes in ARVC patients, performing CA in this population may improve outcomes [18]. Of note, ARVC patients may undergo other invasive procedures (such as electrophysiological studies, voltage mapping-guided biopsies, or CA for ventricular arrhythmias), and CA for atrial arrhythmias may be potentially performed during the same procedure, reducing the need for multiple interventions and increasing the overall procedural benefit. Second, PVI currently represents the gold-standard lesion set for AF management. Despite the pathophysiology of AF being incompletely understood, PVI seems to be not only effective in the general population but also in channelopathies (e.g., Brugada syndrome [19]) and cardiomyopathies (e.g., hypertrophic cardiomyopathy [20]). The observation that PVI provided a good long-term outcome in our study suggests that AF in this population is driven by similar mechanisms as in the general population. Obtaining data regarding the efficacy of CA for atrial arrhythmias in specific subpopulations may provide important knowledge for refining our understanding of both atrial arrhythmias and of the underlying disease itself.

\subsection{Limitations}

There were several limitations to our study. First, given the rarity of patients with ARVC, particularly those presenting with symptomatic AA, the number of patients in- 
cluded in this study is small. Second, given the retrospective nature of the study, it was not possible to uniformly assess echocardiographic imaging criteria and pursue arrhythmic follow-up strategies due to differences in clinical practice of participating centers. Third, cardiac magnetic resonance imaging (CMR) with a specific protocol assessing atrial fibrosis was not available in our cohort. Finally, it should also be noted that all centers involved in the study were third-level referral centers, and, therefore, some degree of selection and referral bias cannot be ruled out. Further studies are needed to confirm generalizability of this data in an all-comer population of patients with ARVC.

\section{Conclusions}

CA represents an effective and safe therapeutic option for the management of atrial arrhythmias in patients with ARVC, with overall long-term success rates comparable to those reported in the general population. Larger prospective studies are needed to confirm these findings.

Author Contributions: Conceptualization, A.G., F.D. and A.M.S.; methodology, A.G. and A.M.S.; software, A.G.; formal analysis, A.G. and A.M.S.; investigation, P.G.P. and A.M.S.; data curation, C.A.J., L.C., N.S., S.K., S.M., P.C., A.M., H.K.J., A.S., S.C., G.B.F., P.G.P., C.T., J.-P.S., A.D.R., F.R., H.C., F.D. and A.M.S.; writing—original draft preparation, A.G. and A.M.S.; writing—review and editing C.A.J., L.C., N.S., S.K., S.M., P.C., A.M., H.K.J., A.S., S.C., G.B.F., M.C., P.G.P., C.T., J.-P.S., A.D.R., F.R., H.C., F.D. and A.M.S.; supervision, H.C., F.D. and A.M.S.; funding acquisition, C.B., F.D. and A.M.S. All authors have read and agreed to the published version of the manuscript.

Funding: The Zurich ARVC Program is supported by generous grants from the Georg and Bertha Schwyzer-Winiker Foundation, Baugarten Foundation, Leonie-Wild Foundation, Swiss Heart Foundation, and Swiss National Science Foundation. H.K.J. is supported by grants from the Novo Nordisk Foundation, Denmark (NNF18OC0031258 and NNF20OC0065151. PGP is supported by the Swedish Heart-Lung Foundation and governmental funding within the Swedish national healthcare system (ALF). The Johsn Hopkins ARVC Program is supported by funding from Fondation Leducq and UL1TR001079 (NCATS) and by the Leonie-Wild Foundation, the Leyla Erkan Family Fund for ARVD Research, the Dr. Francis P. Chiramonte Private Foundation, the Dr. Satish, Rupal, and Robin Shah ARVD Fund at Johns Hopkins, the Bogle Foundation, the Healing Hearts Foundation, the Campanella family, the Patrick J. Harrison Family, the Peter French Memorial Foundation, and the Wilmerding Endowments. This work was performed during A.G.'s tenure as the Wilton W. Webster Fellowship in Clinical Cardiac Catheter Ablation Fellow of the Heart Rhythm Society.

Institutional Review Board Statement: The study was conducted according to the guidelines of the Declaration of Helsinki, and approved by the Zurich cantonal ethical committee (approval number: KEK-ZH Nr. 2014-0443, date 28 August 2014).

Informed Consent Statement: Informed consent was obtained from all subjects if required by the local institutional review board.

Data Availability Statement: Data supporting the current study are available upon reasonable request to the corresponding author.

Acknowledgments: We thank Robert Manka, Departments of Cardiology and Radiology, University Heart Center, Zurich, Switzerland, for performing and interpreting the cardiac MRI scans in the Swiss cohort. We thank Stephan Willems, and Karl-Heinz Kuck, for expert guidance.

Conflicts of Interest: A.M.S. received educational grants through his institution from Abbott, Bayer Healthcare, Biosense Webster, Biotronik, Boston Scientific, BMS/Pfizer, and Medtronic, and speaker fees from Bayer Healthcare, Daiichi-Sankyo, and Novartis. H.K.J. is supported by grants from the Novo Nordisk Foundation, Denmark (NNF18OC0031258 and NNF20OC0065151), and received lecture fees from Abbott Denmark and Biosense Webster, Europe. Platonov is supported by the Swedish Heart-Lung Foundation and governmental funding within the Swedish national healthcare system (ALF). The other authors do not report any conflict of interest. 


\section{References}

1. Corrado, D.; Link, M.S.; Calkins, H. Arrhythmogenic Right Ventricular Cardiomyopathy. N. Engl. J. Med. 2017, 376, 61-72. [CrossRef] [PubMed]

2. Fox, P.R.; Maron, B.J.; Basso, C.; Liu, S.-K.; Thiene, G. Spontaneously Occurring Arrhythmogenic Right Ventricular Cardiomyopathy in the Domestic Cat: A New Animal Model Similar to the Human Disease. Circulation 2000, 102, 1863-1870. [CrossRef] [PubMed]

3. Saguner, A.M.; Ganahl, S.; Kraus, A.; Baldinger, S.H.; Medeiros-Domingo, A.; Saguner, A.R.; Mueller-Burri, S.A.; Wolber, T.; Haegeli, L.M.; Krasniqi, N.; et al. Clinical Role of Atrial Arrhythmias in Patients with Arrhythmogenic Right Ventricular Dysplasia. Circ. J. 2014, 78, 2854-2861. [CrossRef] [PubMed]

4. Zghaib, T.; Bourfiss, M.; van der Heijden, J.F.; Loh, P.; Hauer, R.N.; Tandri, H.; Calkins, H.; Nazarian, S.; te Riele, A.S.J.M.; Zimmerman, S.L.; et al. Atrial Dysfunction in Arrhythmogenic Right Ventricular Cardiomyopathy: Value of Quantitative Magnetic Resonance Analysis in Predicting Atrial Arrhythmias. Circ. Cardiovasc. Imaging 2018, 11, e007344. [CrossRef] [PubMed]

5. Li, G.; Fontaine, G.H.; Fan, S.; Yan, Y.; Bode, P.K.; Duru, F.; Frank, R.; Saguner, A.M. Right atrial pathology in arrhythmogenic right ventricular dysplasia. Cardiol. J. 2019, 26, 736-743. [CrossRef] [PubMed]

6. Platonov, P.G.; Christensen, A.H.; Holmqvist, F.; Carlson, J.; Haunsø, S.; Svendsen, J.H. Abnormal atrial activation is common in patients with arrhythmogenic right ventricular cardiomyopathy. J. Electrocardiol. 2011, 44, 237-241. [CrossRef] [PubMed]

7. Camm, C.F.; James, C.A.; Tichnell, C.; Murray, B.; Bhonsale, A.; te Riele, A.S.J.M.; Judge, D.P.; Tandri, H.; Calkins, H. Prevalence of atrial arrhythmias in arrhythmogenic right ventricular dysplasia/cardiomyopathy. Heart Rhythm 2013, 10, 1661-1668. [CrossRef] [PubMed]

8. Baturova, M.A.; Haugaa, K.H.; Jensen, H.K.; Svensson, A.; Gilljam, T.; Bundgaard, H.; Madsen, T.; Hansen, J.; Chivulescu, M.; Christiansen, M.K.; et al. Atrial fibrillation as a clinical characteristic of arrhythmogenic right ventricular cardiomyopathy: Experience from the Nordic ARVC Registry. Int. J. Cardiol. 2020, 298, 39-43. [CrossRef] [PubMed]

9. Rujirachun, P.; Wattanachayakul, P.; Charoenngam, N.; Winijkul, A.; Ungprasert, P. Prevalence of atrial arrhythmia in patients with arrhythmogenic right ventricular cardiomyopathy: A systematic review and meta-analysis. J. Cardiovasc. Med. 2020. [CrossRef] [PubMed]

10. Brugada, J.; Katritsis, D.G.; Arbelo, E.; Arribas, F.; Bax, J.J.; Blomström-Lundqvist, C.; Calkins, H.; Corrado, D.; Deftereos, S.G.; Diller, G.-P.; et al. 2019 ESC Guidelines for the management of patients with supraventricular tachycardiaThe Task Force for the management of patients with supraventricular tachycardia of the European Society of Cardiology (ESC). Eur. Heart J. 2020, 41, 655-720. [CrossRef] [PubMed]

11. Kirchhof, P.; Benussi, S.; Kotecha, D.; Ahlsson, A.; Atar, D.; Casadei, B.; Castella, M.; Diener, H.-C.; Heidbuchel, H.; Hendriks, J.; et al. 2016 ESC Guidelines for the management of atrial fibrillation developed in collaboration with EACTS. Eur. Heart J. 2016, 37, 2893-2962. [CrossRef] [PubMed]

12. Chu, A.F.; Zado, E.; Marchlinski, F.E. Atrial Arrhythmias in Patients with Arrhythmogenic Right Ventricular Cardiomyopathy/Dysplasia and Ventricular Tachycardia. Am. J. Cardiol. 2010, 106, 720-722. [CrossRef] [PubMed]

13. Cardona-Guarache, R.; Åström-Aneq, M.; Oesterle, A.; Asirvatham, R.; Svetlichnaya, J.; Marcus, G.M.; Gerstenfeld, E.P.; Klein, L.; Scheinman, M.M. Atrial arrhythmias in patients with arrhythmogenic right ventricular cardiomyopathy: Prevalence, echocardiographic predictors, and treatment. J. Cardiovasc. Electrophysiol. 2019, 30, 1801-1810. [CrossRef] [PubMed]

14. Marcus, F.I.; McKenna, W.J.; Sherrill, D.; Basso, C.; Bauce, B.; Bluemke, D.A.; Calkins, H.; Corrado, D.; Cox, M.G.P.J.; Daubert, J.P.; et al. Diagnosis of arrhythmogenic right ventricular cardiomyopathy/dysplasia: Proposed Modification of the Task Force Criteria. Eur. Heart J. 2010, 31, 806-814. [CrossRef] [PubMed]

15. Brouwer, T.F.; Yilmaz, D.; Lindeboom, R.; Buiten, M.S.; Olde Nordkamp, L.R.A.; Schalij, M.J.; Wilde, A.A.; van Erven, L.; Knops, R.E. Long-Term Clinical Outcomes of Subcutaneous versus Transvenous Implantable Defibrillator Therapy. J. Am. Coll. Cardiol. 2016, 68, 2047-2055. [CrossRef] [PubMed]

16. Mazzanti, A.; Ng, K.; Faragli, A.; Maragna, R.; Chiodaroli, E.; Orphanou, N.; Monteforte, N.; Memmi, M.; Gambelli, P.; Novelli, V.; et al. Arrhythmogenic Right Ventricular Cardiomyopathy. J. Am. Coll. Cardiol. 2016, 68, 2540-2550. [CrossRef]

17. Packer, D.L.; Mark, D.B.; Robb, R.A.; Monahan, K.H.; Bahnson, T.D.; Poole, J.E.; Noseworthy, P.A.; Rosenberg, Y.D.; Jeffries, N.; Mitchell, L.B.; et al. Effect of Catheter Ablation vs. Antiarrhythmic Drug Therapy on Mortality, Stroke, Bleeding, and Cardiac Arrest among Patients with Atrial Fibrillation: The CABANA Randomized Clinical Trial. JAMA 2019, 321, 1261. [CrossRef]

18. Müssigbrodt, A.; Knopp, H.; Efimova, E.; Weber, A.; Bertagnolli, L.; Hilbert, S.; Kosiuk, J.; Dinov, B.; Bode, K.; Kircher, S.; et al. Supraventricular arrhythmias in patients with arrhythmogenic right ventricular dysplasia/cardiomyopathy associate with long-term outcome after catheter ablation of ventricular tachycardias. EP Eur. 2018, 20, 1182-1187. [CrossRef] [PubMed]

19. Mugnai, G.; Hünük, B.; Ströker, E.; Ruggiero, D.; Coutino-Moreno, H.E.; Takarada, K.; De Regibus, V.; Choudhury, R.; Abugattas de Torres, J.P.; Moran, D.; et al. Long-term outcome of pulmonary vein isolation in patients with paroxysmal atrial fibrillation and Brugada syndrome. EP Eur. 2018, 20, 548-554. [CrossRef] [PubMed]

20. Zheng, S.; Jiang, W.; Dai, J.; Li, K.; Shi, H.; Wu, W.; Liu, X.; He, B.; Qiu, X.; Song, Z. Five-year outcomes after catheter ablation for atrial fibrillation in patients with hypertrophic cardiomyopathy. J. Cardiovasc. Electrophysiol. 2020, 31, 621-628. [CrossRef] 